\title{
Pattern adaptation and cross-orientation interactions in the primary visual cortex
}

\author{
Matteo Carandini a,b,*, J. Anthony Movshon ${ }^{\mathrm{a}}$, David Ferster ${ }^{\mathrm{b}}$ \\ ${ }^{a}$ Howard Hughes Medical Institute and Center for Neural Science, New York University, 4 Washington Place 809, New York, NY 10003, USA \\ ${ }^{\mathrm{b}}$ Department of Neurobiology and Physiology, Northwestern University, Evanston, IL 60208, USA
}

Accepted 8 April 1998

\begin{abstract}
The responsiveness of neurons in the primary visual cortex (V1) is substantially reduced after a few seconds of visual stimulation with an effective pattern. This phenomenon, called pattern adaptation, is uniquely cortical and is the likely substrate of a variety of perceptual after-effects. While adaptation to a given pattern reduces the responses of V1 neurons to all subsequently viewed test patterns, this reduction shows some specificity, being strongest when the adapting and test patterns are identical. This specificity may indicate that adaptation affects the interaction between groups of neurons that are jointly activated by the adapting stimulus. We investigated this possibility by studying the effects of adaptation to visual patterns containing one or both of two orientations - the preferred orientation for a cell, and the orientation orthogonal to it. Because neurons in the primary visual cortex are sharply tuned for orientation, stimulation with orthogonal orientations excites two largely distinct populations of neurons. With intracellular recordings of the membrane potential of cat V1 neurons, we found that adaptation to the orthogonal orientation alone does not evoke the hyperpolarization that is typical of adaptation to the preferred orientation. With extracellular recordings of the firing rate of macaque V1 neurons, we found that the responses were not reduced by adaptation to the orthogonal orientation alone nearly as much as by adaptation to the preferred orientation. In the macaque we also studied the effects of adaptation to plaids containing both the preferred and the orthogonal orientations. We found that adaptation to these stimuli could modify the interactions between orientations. It increased the amount of cross-orientation suppression displayed by some cells, even turning some cells that showed cross-orientation facilitation when adapted to a blank stimulus into cells that show cross-orientation suppression. This result suggests that pattern adaptation can affect the interaction between the groups of neurons tuned to the orthogonal orientations, either by increasing their mutual inhibition or by decreasing their mutual excitation. (C) 1998 Elsevier Science Ltd. All rights reserved.
\end{abstract}

Keywords: Cortex; Adaptation; Hyperpolarization; Orientation; Contrast; After-effects

\section{Introduction}

Thanks to a sophisticated mechanism of light adaptation, the signal transmitted from the retina to the rest of the brain is largely independent of the overall light intensity. Rather than depending on the luminance present in a visual scene, this signal grows with the visual contrast, which is the local variation in luminance relative to the overall mean luminance (Shapley and Enroth-Cugell, 1984). Light adaptation allows the subsequent stages of visual processing to concentrate on various attributes of the visual patterns, with little

* Corresponding author. e-mail: matteo@cns.nyu.edu. concern for the overall illumination, and ultimately allows one to see equally well under a wide range of illumination conditions.

A different mechanism of visual adaptation makes its first appearance in the primary visual cortex (V1). The responses of V1 neurons - which are selective for visual attributes such as position, shape, orientation, direction and speed-are far from constant over time, being substantially reduced after a few seconds of stimulation (Maffei et al., 1973). This reduction is known as pattern adaptation or as contrast adaptation (to distinguish it from light adaptation), and is uniquely cortical. Indeed it is essentially absent in subcortical neurons (Ohzawa et al., 1985; Shou et al., 1996), and it can be elicited through either eye even when the cortical neurons 
receive subcortical input from only one eye (Maffei et al., 1986).

Pattern adaptation in V1 is most likely responsible for a variety of after-effects that can influence the perception of stimulus size or orientation (Blakemore and Campbell, 1968; Gilinsky, 1968; Pantle and Sekuler, 1968), or other stimulus attributes (see review in Graham, 1989). A classical demonstration of an after-effect in the orientation domain is illustrated in Fig. 1, derived from work by Blakemore and colleagues. After about $30 \mathrm{~s}$ of exposure to the two tilted gratings on the left, inspection of the two vertical gratings on the right results in the illusory perception of tilt in the directions opposite to the ones observed during the adaptation period.

The traditional explanation for these perceptual after-effects is essentially based on two assumptions: (1) perception is the result of a weighted sum of the outputs of sensory neurons; and (2) prolonged stimulation with a visual pattern 'fatigues' those sensory neurons that are best tuned to that pattern (Graham, 1989). After prolonged stimulation with a certain pattern, perception would be biased away from the pattern, because the neurons tuned to the pattern respond less strongly than they would in normal conditions (Blakemore and Campbell, 1968).

A difficulty with the fatigue explanation comes from the physiology of V1 neurons. Movshon and Lennie (1979) reported two decades ago that adaptation to a spatial frequency on one flank of a neuron's tuning curve depresses the responses to test stimuli on that flank more than on the other flank. The effects of adaptation on V1 cells are thus not the same for all test

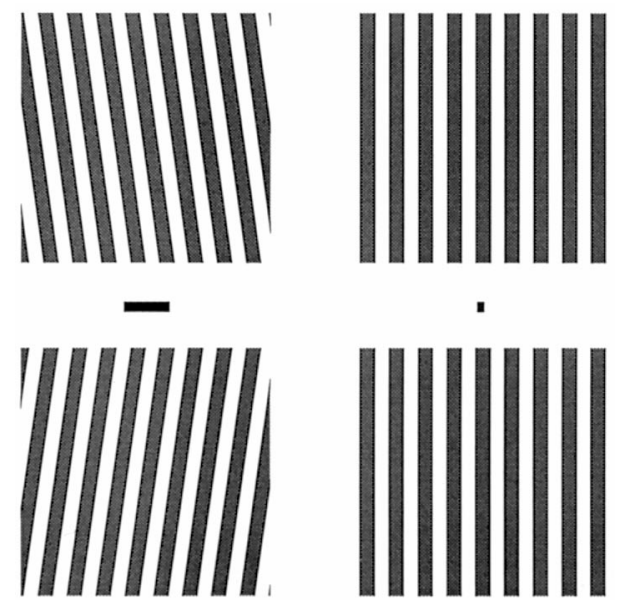

Fig. 1. A classical demonstration of after-effect in the orientation domain, derived from work by Blakemore and colleagues. For about $30 \mathrm{~s}$, look at the line between the two gratings on the left, while moving your eyes from one end of the line to the other (to avoid the formation of retinal after-images). Then, look at the dot between the two gratings on the right. The gratings should briefly appear tilted in the opposite ways of the two gratings on the left. stimuli. Indeed, there is now a large literature (partly cited in Section 4) indicating that adaptation can affect the tuning of the cells for various stimulus parameters. This literature can be summarized as follows. First, a pattern presented in a cell's receptive field has to be effective in driving the cell in order to elicit adaptation. Second, adaptation to a given pattern reduces the responses to all subsequently viewed test patterns. Third, this reduction shows some specificity, being strongest when the adapting and test patterns are identical.

An alternative to the fatigue hypothesis is the theory of adaptation to contingencies proposed by Barlow and Földiák (1989), Barlow, 1990, 1997). According to this theory, cortical neurons would adapt selectively to contingencies in the pattern of activity they receive, through a mechanism that increases the amount of mutual inhibition (or decreases the amount of mutual excitation) between cells that are simultaneously active. This mechanism would explain the specificity of adaptation observed in single neurons; the specificity would result from the simultaneous activity of the tested neuron and of a population of other neurons, the population varying with the adapting stimulus. In principle, this mechanism of adaptation to contingencies could also explain a variety of perceptual phenomena (Barlow and Földiák, 1989; Barlow, 1990, 1997).

To investigate whether adaptation affects the interaction between specific groups of neurons that are jointly activated by the adapting stimulus, we studied the effect of adaptation to visual patterns that contain one or both of two orthogonal orientations. Because neurons in V1 are sharply tuned for orientation, orthogonal orientations are processed by groups of neurons that are essentially nonoverlapping (De Valois et al., 1979; Movshon et al., 1986; Gizzi et al., 1990; Bonhoeffer and Grinvald, 1991). There is however evidence for mutual interactions between the two groups and these interactions are considered to be largely inhibitory (Morrone et al., 1982; Bonds, 1989; Heeger, 1992; Carandini et al., 1997b). It is not known if these interactions can be modified by adaptation to visual patterns.

Our results can be summarized as follows. First, adaptation to a stimulus whose orientation is appropriate for driving a cat V1 neuron causes a hyperpolarization of the neuron's membrane, while adaptation to an orthogonal stimulus does not cause such a hyperpolarization. Second, adaptation to the orthogonal orientation reduces the firing rate responses of macaque V1 neurons much less than adaptation to the preferred grating alone or to the plaid obtained by summing the preferred and orthogonal gratings. Third, adaptation to the plaid containing both orientations can increase the amount of cross-orientation suppression in macaque $\mathrm{V} 1$, to the point of turning some neurons that show cross-orientation facilitation into neurons that show cross-orientation suppression. 
These results indicate that while adaptation to the individual orientations mainly affects separate groups of neurons, both in the cat and in the monkey, adaptation to the two orientations together can affect the interactions between the two groups. In Section 4 we will speculate that pattern adaptation is the result of two mechanisms. One of these mechanisms is in its effects akin to fatigue; it is based on a tonic hyperpolarization and decreases the responsiveness of a cell to all visual stimuli. The other is as hypothesized by Barlow and Földiák (1989); it is based on an increase in mutual inhibition (or a decrease in mutual excitation) between groups of cells, and decreases the responsiveness of those cells to particular stimulus configurations that are similar to the one used to induce adaptation.

\section{Methods}

The data in this paper were obtained in two recent research projects on pattern adaptation in V1. The first project involved intracellular recordings from the cat cortex and was aimed at uncovering the biophysical basis of adaptation (Carandini and Ferster, 1997). The second project involved extracellular recordings in the macaque cortex and studied the effects of adaptation to stimuli containing multiple orientations (Carandini et al., 1997a). The paper presents some previously unpublished results from the first study and a novel analysis of the data in the second study. The methods for the two studies are summarized here for reference.

The effects of adaptation were studied by measuring the responses of visual cortical cells to test stimuli of different contrasts (presented in random order), while the cells were in different states of adaptation. The state of adaptation was controlled by presenting the adapting stimulus first in a long continuous exposure (20-30 s), and then for brief (4s) 'top-up' exposures between test stimuli (Movshon and Lennie, 1979). Stimuli were sinusoidal gratings drifting at $2-6 \mathrm{~Hz}$, or plaids obtained by summing two gratings. Stimuli lasted $4 \mathrm{~s}$, were presented monocularly and had the same mean luminance.

\subsection{Intracellular}

Whole-cell patch recordings in current clamp mode were obtained from barbiturate-anaesthetized, paralyzed adult cats (Carandini and Ferster, 1997; Jagadeesh et al., 1997). Stimuli were presented on a gamma-corrected oscilloscope (mean luminance $15 \mathrm{~cd} /$ $\mathrm{m}^{2}$ ) using an image generator (Innisfree, Cambridge, MA). Adapting stimuli were: (1) a $47 \%$ contrast grating with the preferred orientation for the cell under study; (2) a $47 \%$ contrast grating with the orthogonal orientation; (3) a very low (1.5\%) contrast grating with the preferred orientation (to which is loosely referred to as a 'blank'). Only one type of test stimulus was used, the 'preferred' grating. Blocks with different adapting stimuli were alternated, and each block was run at least three times.

\subsection{Extracellular}

Single-cell extracellular recordings were obtained from opiate-anesthetized, paralyzed adult macaque monkeys (Levitt et al., 1994; Carandini et al., 1997a). Stimuli were generated by a Truevision ATVista graphics controller $(752 \times 582$ pixels, $107 \mathrm{~Hz})$ and displayed on a gamma-corrected Nanao T560i monitor (mean luminance $72 \mathrm{~cd} / \mathrm{m}^{2}$ ). Adapting stimuli were: (1) a blank screen; (2) a grating with the preferred orientation for the cell under study; (3) a grating with the orthogonal orientation; and (4) the plaid obtained by summing the two gratings. The contrast of the adapting stimuli was 25 or $50 \%$ for each component. Non-blank adaptation conditions were arranged in arbitrary order, and each was preceded by a blank adaptation condition and followed by rest periods (12-20 min of blank screen stimulation) and by a blank adaptation condition to ensure that recovery was complete. Test stimuli were the grating with the preferred orientation and the plaid obtained by summing to it the orthogonal grating. The contrast responses to these stimuli were measured three to four times for each adaptation condition.

\section{Results}

We first present data on the membrane potential responses of cat V1, comparing the effect of adaptation to the preferred orientation with those of adaptation to an orthogonal orientation. Then we present data from the firing rate responses of macaque V1, comparing the effects of adaptation to the preferred orientation, to the orthogonal orientation, and to a plaid containing both orientations.

\subsection{Adaptation and hyperpolarization}

The firing rate responses of a typical cat simple cell to drifting gratings of different contrasts are illustrated in Fig. 2A. These responses were obtained with an optimal grating when the cell was adapted to a $1.5 \%$ contrast grating. Because this contrast is quite low (even for cat cells), it is here loosely referred to as a 'blank'. The responses were essentially unaffected by adaptation to a high contrast grating orthogonal to the cell's preferred orientation (Fig. 2B). The responses were instead substantially reduced by adaptation to a high contrast grating with the preferred orientation (Fig. 2C). More contrast was needed to reach a given response in this condition than in the first two conditions. 


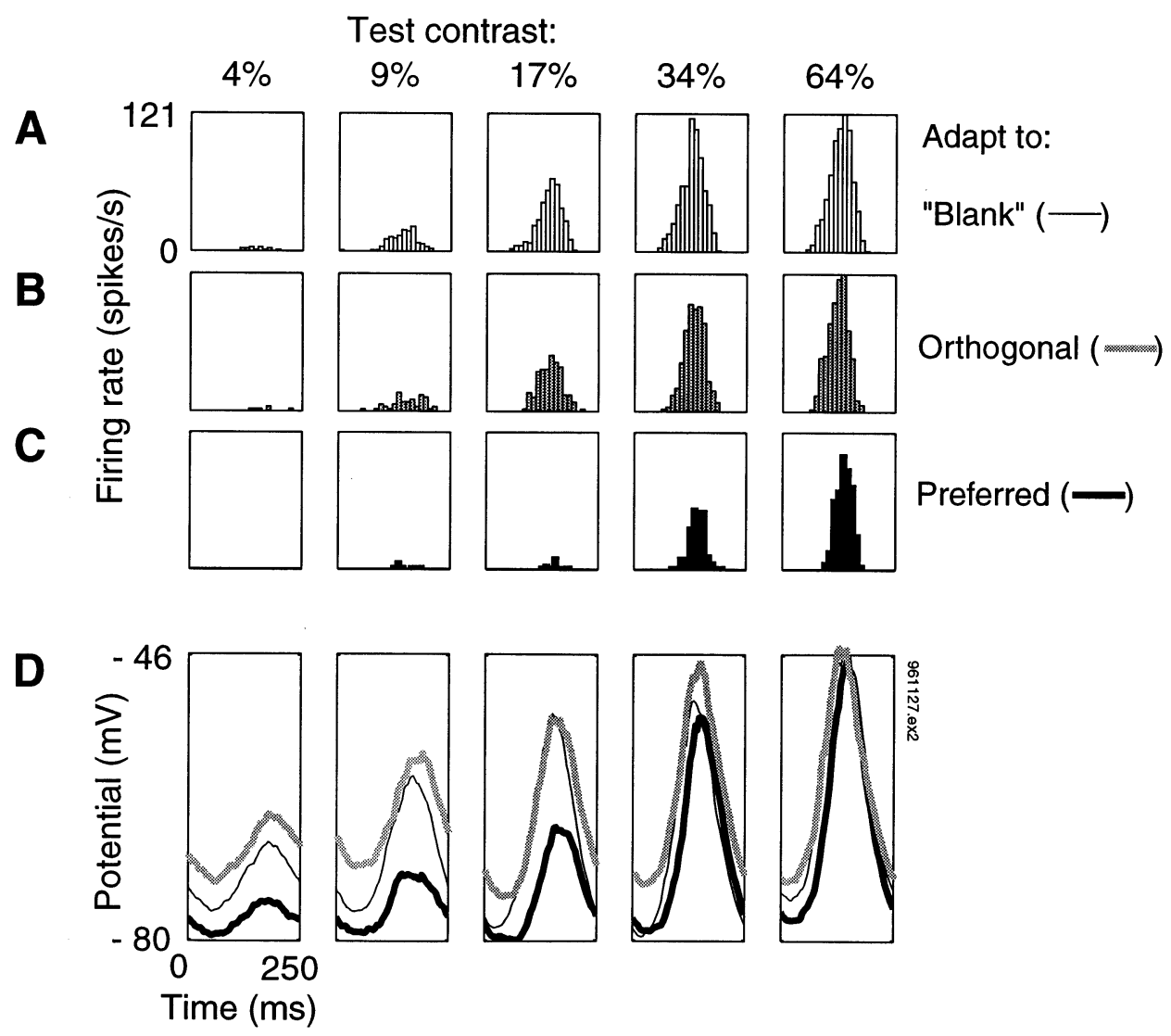

Fig. 2. Intracellularly recorded responses of a cat simple cell to optimal drifting gratings presented at six different test contrasts in three different adaptation conditions. A-C, Period histograms of the spike responses after adaptation to: (A) the 'blank' stimulus (Section 2); (B) a grating oriented orthogonal to the preferred, and (C) a grating with the preferred orientation. D, Cycle averages of the membrane potential after adaptation to the three stimuli. Thin traces are adapted to 'blank' stimulus, thick traces adapted to preferred (black) and orthogonal (gray) gratings.

The membrane potential responses underlying these firing rate responses are illustrated in Fig. 2D. Simple cells respond to drifting sinusoidal gratings with membrane potential fluctuations that are nearly sinusoidal (Jagadeesh et al., 1997). The effects of adaptation on the membrane potential responses can be seen by comparing the thin traces obtained with adaptation to the 'blank' to the thick traces obtained with adaptation to the high contrast gratings. The main effect of adaptation with the grating of preferred orientation (thick black traces) was to hyperpolarize the cell, shifting each trace down by $1-8 \mathrm{mV}$. Adaptation with the grating of orthogonal orientation (thick gray traces) did not hyperpolarize the cell. At some test contrasts it actually caused a depolarization, shifting the traces up by $2-3$ $\mathrm{mV}$.

The effects of pattern adaptation on the responses of a V1 cell can be summarized by plotting some response measures derived from the firing rate and from the membrane potential against the stimulus contrast. For the cell in Fig. 2, these contrast responses are illustrated in Fig. 3.
The contrast responses measured during adaptation to the 'blank' stimulus are indicated by open circles. The contrast response measured from the mean firing rate (Fig. 3A) is typical of $\mathrm{V} 1$ cells, being sigmoidal in shape, valued close to zero for very low test contrasts, and almost constant for high test contrasts. Increasing test contrast had two main effects on the membrane potential of the cell. First, it increased the size of the stimulus-driven membrane potential modulation (Fig. $3 \mathrm{~B}$ ), which is the amplitude of a $4 \mathrm{~Hz}$ sinusoid fitted to the responses in Fig. 2D. This quantity grows from about $1 \mathrm{mV}$ at $2 \%$ contrast to almost $15 \mathrm{mV}$ at $64 \%$ contrast. Second, it increased the mean membrane potential (Fig. 3C) by around $5 \mathrm{mV}$, from around -70 $\mathrm{mV}$ to around $-65 \mathrm{mV}$.

The effects of adaptation to the grating with preferred orientation are illustrated by the filled triangles. Adaptation to this stimulus substantially reduced the firing rate responses, shifting them so that higher contrasts were needed to obtain comparable responses. This effect is entirely in line with previous findings (Movshon and Lennie, 1979; Ohzawa et al., 1982; 
Albrecht et al., 1984; Ohzawa et al., 1985; Sclar et al., 1989). The reduction in firing rate responses is accompanied by a reduction in the size of the stimulus-driven membrane potential modulation at intermediate test contrasts (Fig. 3B) and in the mean membrane potential at all but the highest test contrasts (Fig. 3C). The hyperpolarization caused by adaptation to a grating with preferred orientation is typical of simple cells, and has been reported before (Carandini and Ferster, 1997).

By contrast, the effects of adaptation to the orthogonal grating (filled squares) were minor. The firing rate was essentially unchanged (Fig. 3A), and so was the
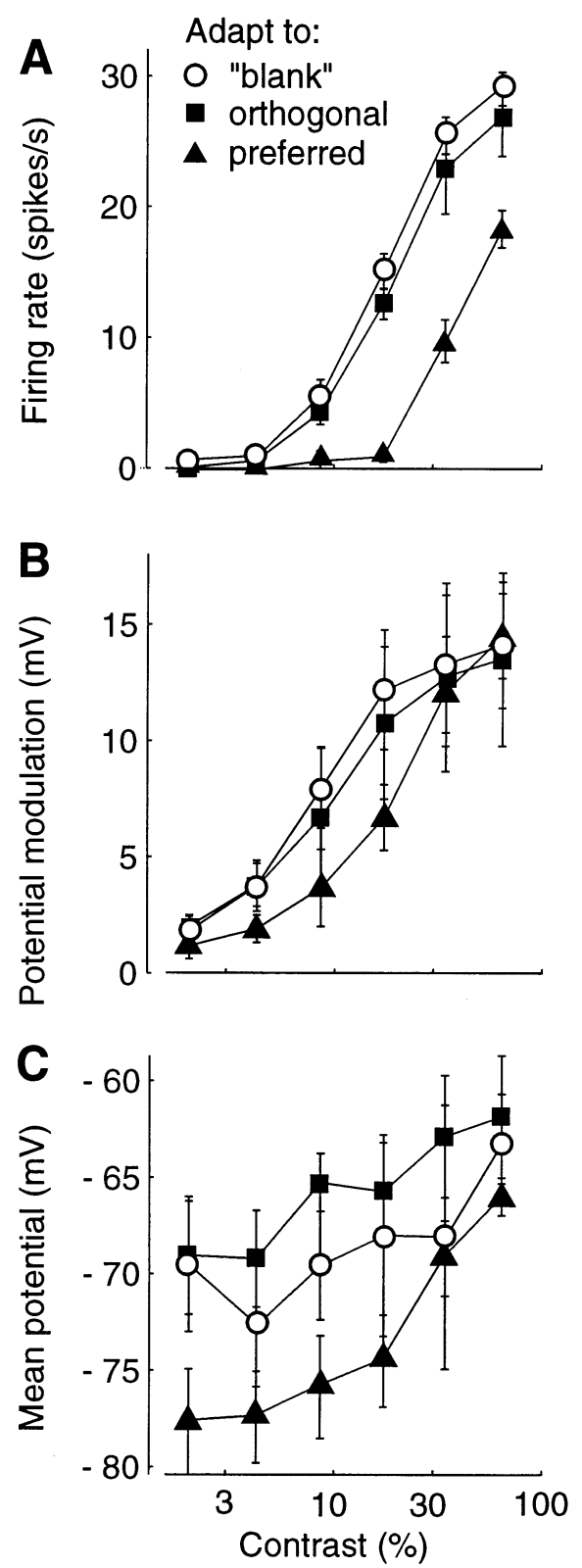

Fig. 3. Contrast response curves derived from the data in Fig. 2. A, The mean firing rate response. $\mathrm{B}$, The modulation of the membrane potential at the stimulus frequency; $\mathrm{C}$, The mean membrane potential. Error bars are twice the standard error of the mean over different blocks. size of the stimulus-driven membrane potential modulation (Fig. 3B). Adaptation to the orthogonal grating did not hyperpolarize the membrane: On the contrary, it depolarized the membrane slightly increasing the mean membrane potential (Fig. 3C).

The lack of hyperpolarization caused by adaptation to an orthogonal grating was consistently displayed by all eight cells tested this way. Data from four of these cells (in addition to the one in Figs. 2 and 3) are illustrated in Fig. 4. All of these cells displayed a clear hyperpolarization when adapted to the grating with the preferred orientation (triangles). None of the cells displayed hyperpolarization when adapted to the orthogonal grating (squares). Instead, two of them displayed a slight depolarization similar to that of the cell in Figs. 2 and 3 . The remaining three cells in the sample (not shown, two complex, one simple) gave noisier responses and did not exhibit clear signs of adaptation even to the preferred stimulus.

In simple cells, the hyperpolarization caused by adaptation to the grating with preferred orientation is larger if the subsequent test stimulus has low contrast than if it has high contrast. This effect can be observed in both of the simple cells presented here, in Fig. 3C and Fig. 4A, and is commonly observed in simple cells but not in complex cells (Carandini and Ferster, 1997). Because the hyperpolarization in simple cells tends to be largest for test contrasts approaching zero, it is essentially tonic, independent of visual stimulation after the presentation of the adapting stimulus.

\subsection{Adaptation and cross-orientation suppression}

We have found that the orthogonal grating alone is generally not effective in eliciting adaptation. Does its presentation as an adapting stimulus affect the subsequent responses of a cell in any way? To answer this question we investigated the effects of adaptation to the plaid obtained by summing the two gratings. These effects were studied as part of an experiment (Carandini et al., 1997a) aimed at testing the theory of adaptation to contingencies proposed by Barlow and Földiák, which was mentioned in the Introduction. We measured the firing rate responses of $\mathrm{V} 1$ cells in the macaque to two different stimuli; a grating with the preferred orientation and the plaid obtained by summing an orthogonal grating to it. We subsequently adapted the cells to one of three stimuli in turn, the preferred grating, the orthogonal grating, and the plaid, and observed how this adaptation affected the contrast responses.

An example of the results of these experiments is illustrated in Fig. 5. The cell is a complex cell, and when adapted to the blank stimulus (Fig. 5A) gave robust responses to both the preferred grating (open symbols) and the plaid obtained by summing an or- 


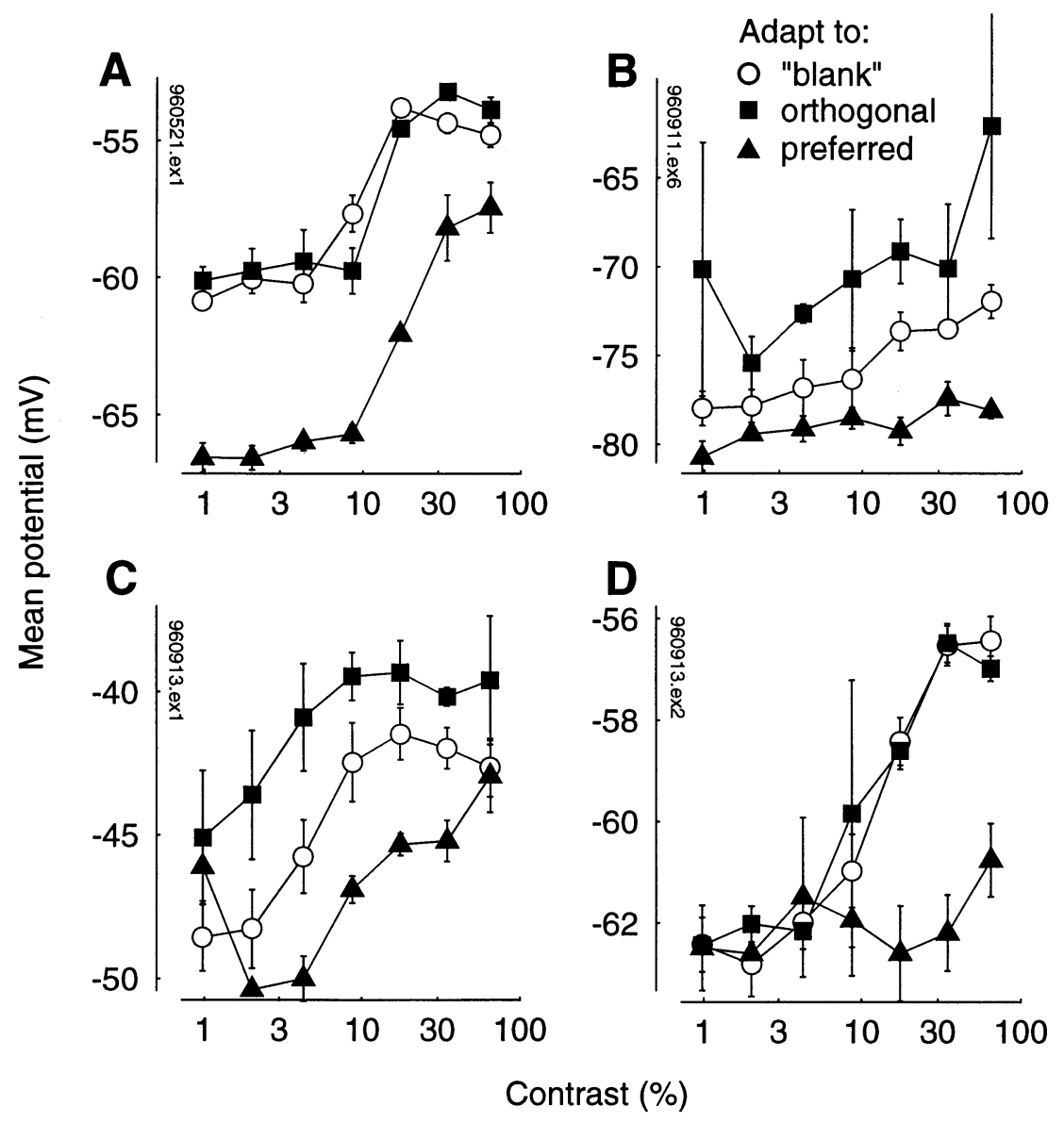

Fig. 4. Contrast response curves for the mean membrane potential of four cells in cat V1, in three different adaptation conditions. Cell in A was simple, others were complex.

thogonal grating to it (filled symbols). After adaptation to the preferred grating (Fig. 5B), both responses were substantially reduced. Adaptation to the orthogonal grating (Fig. 5C) had a much milder effect, consistent with the previous intracellular findings. Adaptation to the plaid (Fig. 5D) instead had a strong effect, stronger on the responses to the plaid (filled symbols) than on the responses to the preferred grating (open symbols).

In an effort to describe the strength of adaptation with a single measure, an adaptation index was considered (Carandini et al., 1997a). This index is the ratio between the sum of the unadapted responses to a given test and the sum of the adapted responses to the same test, these sums being computed over all different test contrasts. In geometric terms, the index is the ratio of the areas under the unadapted and adapted contrast response curves. For example, for the cell in Fig. 5, the adaptation index for a plaid adaptor measured with grating stimuli is given by the area under the open symbols in Fig. 5A divided by the area under the open symbols in Fig. 5D. The areas were computed from hyperbolic-ratio curves fitted to the data (Albrecht and Hamilton, 1982; Albrecht et al., 1984; Sclar et al., 1989), so that one could compare adaptation conditions even if when the test contrasts used were different. Bootstrap estimates (Efron and Tibshirani, 1991) were performed to obtain means and standard deviations for the measurements. For this purpose, the data obtained in individual blocks of test stimuli were treated as independent random variables.

The adaptation indices obtained with the three types of adapting stimulus and the two types of test stimulus for the sample of eight cells that were tested in the previous study (Carandini et al., 1997a) are illustrated in Fig. 6. The adaptation indices were all greater than one, implying that adaptation always reduced the responses. Fig. 6 (panel A) shows that the data in this study agree with the data obtained intracellularly in the cat. The effects of adaptation to the preferred grating tended to be much stronger than those of adaptation to the orthogonal grating. This was the case for both types of test stimuli, the preferred grating (open symbols), and the plaid (closed symbols).

In Fig. 6 (panel B) the effect of adaptation to the plaid and to the preferred grating is compared. Because the plaid is the sum of the preferred grating and of an orthogonal grating, its stimulus energy is higher. If the strength of adaptation were a simple monotonic func- 

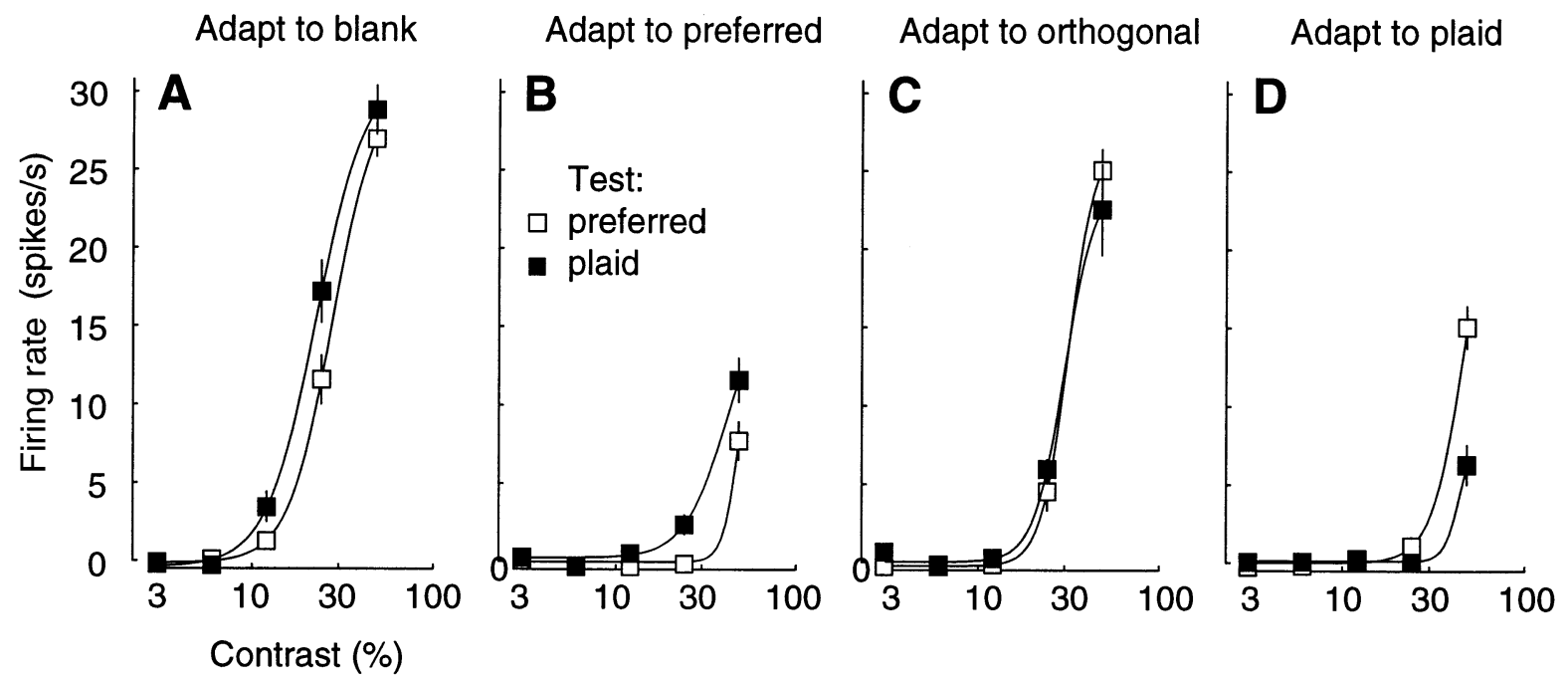

Fig. 5. Contrast response curves measured from a macaque complex cell in four different adaptation conditions. The test stimuli were the preferred grating (open symbols) and the plaid obtained by summing to it an orthogonal grating (filled symbols). Adapting stimuli were a blank screen (A), the preferred grating (B) a grating orthogonal to it (C) and the plaid (D). The ordinates represent the mean firing rate response. Error bars are twice the standard error of the mean over different stimulus presentations. The curves fitted to the data are standard hyperbolic ratio functions (see text).

tion of the stimulus energy, it would be higher for the plaid than for the preferred grating, and the data points would occupy the area to the left of the diagonal identity line. Instead, it was found that the strength of adaptation tended to depend on the stimulus used: in many cases, the responses were reduced more by adaptation to the preferred grating (abscissa) than by adaptation to the plaid (ordinate).

While adaptation to the plaid was not always the strongest condition, it had an effect on the cells that was not shared by adaptation to the individual components; it tended to increase the amount of cross-orientation suppression shown by the cells, and it even turned some cells that showed cross-orientation facilitation into cells that showed cross-orientation suppression. For example, when adapted to the blank stimulus the cell in Fig. 5 showed cross-orientation facilitation: it gave responses to the plaid that were slightly but significantly larger than the responses to the grating (Fig. $5 \mathrm{~A})$. This was still the case also after adaptation to the preferred grating (Fig. 5B). When adapted to the plaid, instead, the cell showed cross-orientation suppression; it gave responses to the plaid that were smaller than the responses to the grating $(5 \mathrm{D})$.

To study whether this effect was shared by other cells in the sample, we defined an index of cross-orientation interaction, which bears close resemblance to the adaptation index. The index of cross-orientation interaction is the ratio between the sum of the responses to the plaid and the sum of the responses to the preferred grating, these sums being computed over all different test contrasts. For example, for the cell in Fig. 5, the index of cross-orientation interaction measured during adaptation to a given stimulus is given by the area under the closed symbols divided by the area under the open symbols. The index is greater than one when there is cross-orientation suppression, i.e. adding an orthogonal grating to the preferred grating reduces the responses. It is smaller than one when there is cross-orientation facilitation, i.e. adding an orthogonal grating to the preferred grating increases the responses.

Using the index of cross-orientation interaction, it was found that in five of the cells (out of eight) crossorientation suppression was stronger after adaptation to the plaid than after adaptation to a blank stimulus. This is illustrated in Fig. 7, where the abscissa represents the index when the cells are adapted to the blank stimulus, and the ordinate represents the index when the cells are adapted to the plaid (Fig. 7A), to the orthogonal grating (Fig. 7B), and to the preferred grating (Fig. 7C). Points that lie to the left of the diagonal identity line correspond to cells in which cross-orientation suppression was stronger when adapted to one of the visual patterns than when adapted to the blank stimulus. In panel A, corresponding to adaptation to the plaid stimulus, there are five points that lie in this region, and the remaining three points lie on the identity line. While the sample is much too small to derive conclusions about neuronal populations, the error bars associated with our measurements are generally small, and allow one to make statements about the individual cells. In particular, the figure shows that for three cells adaptation to the plaid changed the sign of the interaction between orientations. The index of cross-orientation interaction for these cells changed from being less than one when 
adapted to the blank (indicating facilitation) to being more than one when adapted to the plaid (indicating suppression).

These effects were not obtained by adaptation to either the preferred or the orthogonal gratings alone. In the six cells that were tested during adaptation to the orthogonal grating (Fig. 7B) the points either lie on the identity line or to the right of it, indicating that crossorientation suppression was either unaffected by adaptation, or it was actually reduced. The effects of adaptation to the preferred grating (Fig. 7C) on the degree of cross-orientation suppression were noisier, with many of the error bars approaching or touching

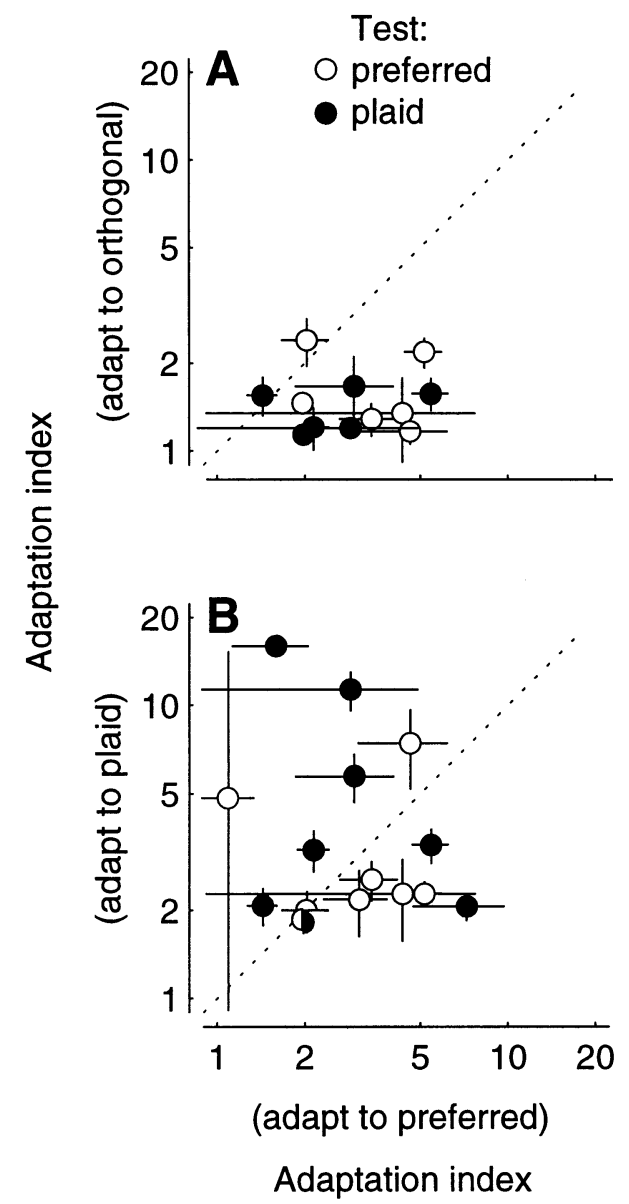

Fig. 6. Strength of adaptation to the preferred grating, the orthogonal grating, and the plaid, measured in eight cells in macaque. V1. Data from Carandini et al. (1997a). For each cell, the adaptation index is the ratio between the sum of the unadapted responses to a given test and the sum of the adapted responses to the same test, with the sums being computed over all different test contrasts. Abscissas represent the adaptation index when adapted to the preferred grating, ordinates represent adaptation index obtained with adaptation to the orthogonal grating (A) and with the plaid obtained by summing the two gratings (B). Indices were measured with preferred grating (open symbols) and plaid (filled symbols) as a test stimulus. Semicircles are used when two symbols overlap. Error bars indicate two standard deviations (bootstrap estimates). Two cells were not adapted to the orthogonal grating, and do not appear in A. Diagonal lines indicate the identity.
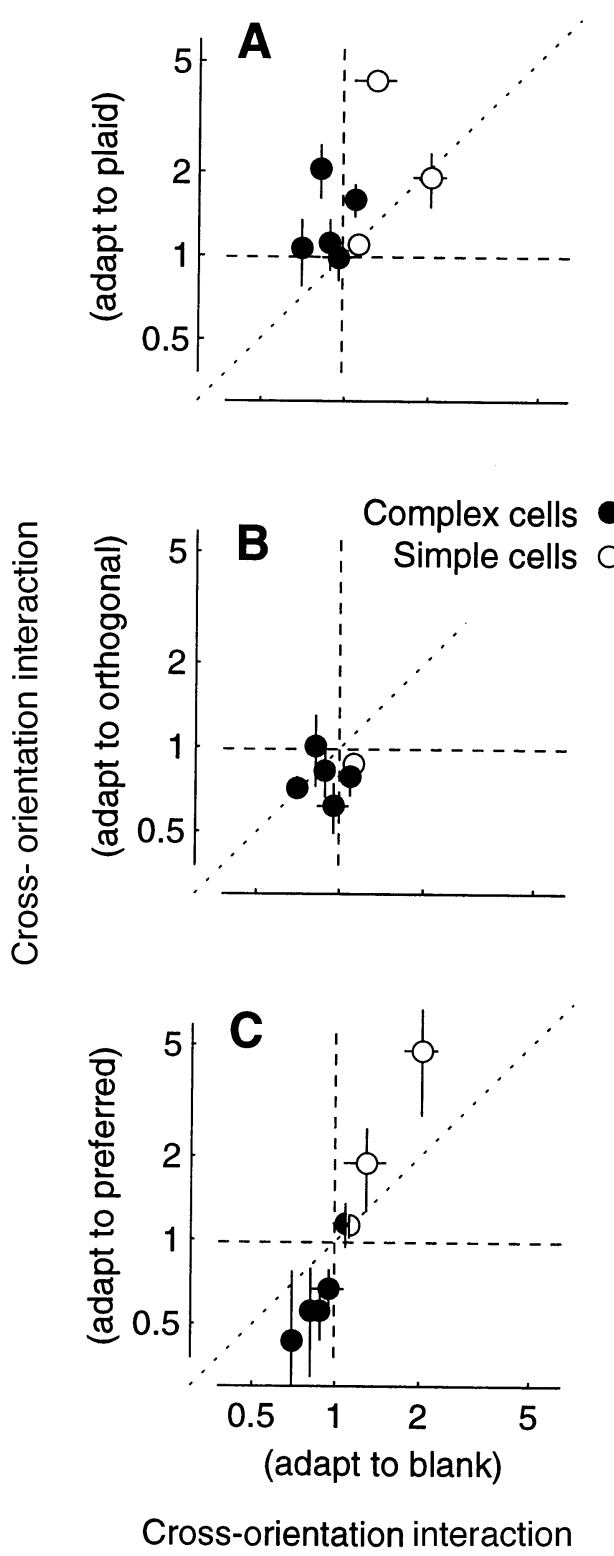

Fig. 7. Effect of adaptation on cross-orientation interactions. Same data as Fig. 6, from Carandini et al. (1997a). For each cell, given an adaptation condition, the cross-orientation interaction index is the ratio between the sum of the responses to the plaid and the sum of the responses to the grating, with the sums being computed over all different test contrasts. Values above one indicate cross-orientation suppression, and values below one indicate cross-orientation facilitation. Abscissa indicates the cross-orientation interaction index when adapted to the blank, ordinates indicate cross-orientation interaction index obtained with adaptation to the plaid (A), to the orthogonal grating alone (B), and to the preferred grating alone $(\mathrm{C})$. Open symbols indicate simple cells, filled symbols indicate simple cells. Semicircles are used when two symbols overlap. Error bars indicate two standard deviations (bootstrap estimates). Two cells were not adapted to the orthogonal grating, and do not appear in B. Diagonal lines indicate the identity.

the diagonal identity line. Overall, these effects were mixed; cross-orientation suppression was increased in four cells, unchanged in two and decreased in the 
remaining two. In no case however did adaptation to the preferred grating change the sign of the interaction between orientations. For example, cells that showed cross-orientation facilitation when adapted to the blank stimulus showed similar or increased cross-orientation facilitation when adapted to the preferred grating.

\section{Discussion}

Neurons in the primary visual cortex are sharply tuned for orientation, to the point of being largely unresponsive to orientations that are orthogonal to their preferred orientation. There are however interactions between stimuli of different orientations, and the response to the preferred orientation can be modified by adding an orthogonal grating to the stimulus to form a plaid (Morrone et al., 1982; Bonds, 1989; Heeger, 1992; Carandini et al., 1997b). Stimulation with the plaid stimulus excites two populations of neurons at the same time, tuned to orthogonal orientations (De Valois et al., 1979; Movshon et al., 1986; Gizzi et al., 1990; Bonhoeffer and Grinvald, 1991). We have studied the effects of prolonged stimulation with patterns containing the orthogonal orientation, and compared them with previously known effects of pattern adaptation. First, we found that whereas adaptation to the preferred orientation causes a hyperpolarization in the membrane potential of the neurons (Carandini and Ferster, 1997), adaptation to the orthogonal orientation alone does not cause such a hyperpolarization. Second, we have shown a similar effect in the firing rate responses of monkey V1 neurons, which were not reduced by adaptation to the orthogonal orientation alone nearly as much as by adaptation to the preferred orientation. Third, we have shown that adaptation to plaids containing both the preferred and the orthogonal orientations can increase the amount of cross-orientation suppression displayed by a cell, turning some cells that showed cross-orientation facilitation when adapted to a blank stimulus into cells that show cross-orientation suppression.

The scarce effects of adaptation to the orthogonal orientation that we observed in the membrane potential of cat V1 neurons and in the firing rate of monkey V1 neurons are consistent with previous measurements of the firing rate of cat V1 neurons. Vautin and Berkeley (1977) found no effect of adaptation with a grating orthogonal to the preferred orientation on the firing rate responses to a grating drifting in the preferred direction. Hammond et al. (1989) systematically varied the orientation of the adapting stimulus and found that the reduction in the responses was maximal when the adapting grating was oriented in the cell's preferred orientation. Finally, Allison and Martin (1997) studied the effect of adaptation to a variety of gratings on the contrast responses measured with preferred gratings, and found that it was weakest when the adapting grating was orthogonal to the preferred grating.

Vautin and Berkeley (1977) suggested that the magnitude of the reduction in response caused by different adapting stimuli depends on the degree to which these stimuli are effective at inducing a response. The strength of adaptation is however now known to depend not only on the adapting stimulus, but also on the test stimulus used to measure the responses after adaptation. In general, adaptation reduces the responses to all subsequently presented stimuli, but it is strongest when the test and adapting stimuli are matched. This specificity of adaptation has been observed in the spatial frequency domain (Movshon and Lennie, 1979; Saul and Cynader, 1989a), in the orientation domain (Marlin et al., 1988; Hammond et al., 1989; Saul and Cynader, 1989a; Giaschi et al., 1993), and in the temporal frequency domain (Saul and Cynader, 1989b). The results in monkey $\mathrm{V} 1$ also provide some evidence of specificity (Carandini et al., 1997a).

The intracellular results in cat V1 do not address directly the effects of adaptation on different patterns, because the test stimulus was always the same, a grating drifting in the preferred orientation. Because we varied the contrast of this test stimulus, however, we were able to study the effects of adaptation as the contrast of the test stimulus approaches zero. In this respect, we found that in simple cells the hyperpolarization caused by adaptation was tonic, being strongest when the test stimulus had the lowest contrast (Carandini and Ferster, 1997). Because a tonic hyperpolarization has the same effect on the responses to any visual stimulus, and the strength of adaptation is known to depend on the particular combination of adapting and test patterns, the tonic hyperpolarization cannot be the sole mechanism underlying pattern adaptation.

Our results with adaptation to plaids provide some clues as to what the additional mechanism or mechanisms underlying pattern adaptation could be. The fact that cross-orientation suppression in some cells is increased by adaptation to the plaid suggests that adaptation affects the interactions between groups of cells tuned to the two orientations. These interactions become more inhibitory or less excitatory after prolonged stimulation with a pattern, the plaid, that evokes activity in both groups.

It becomes thus tempting to propose that pattern adaptation in the primary visual cortex is the result of two mechanisms, one based on a tonic hyperpolarization and the other based on a modification of the connections between specific groups of cortical cells. The effects of the first mechanism would depend only on the pattern used to induce the adaptation, being strongest if the adapting stimulus is optimal in driving the cell, and would decrease the responsiveness of a cell 
to all visual stimuli. The second mechanism would further decrease the responsiveness of the cell to particular test patterns that are similar to the one used to induce adaptation (Barlow and Földiák, 1989; Barlow, 1990, 1997). A similar double adaptation mechanism was proposed by Földiák (1990) on theoretical grounds, its main motivation being that it would yield a representation of stored patterns that is sparse, i.e. carried by a limited number of neurons.

In principle, both mechanisms could operate either through a decrease in synaptic excitation or through an increase in synaptic inhibition. Indeed, each orientation column in the primary visual cortex sends both inhibitory and excitatory signals to all other orientation columns (Kisvárday and Eysel, 1993; Kisvárday et al., 1994, 1997). There is, however, evidence suggesting that adaptation is caused by a decrease in the excitation received by a cortical cell rather than by an increase in inhibition. Evidence in favor of this hypothesis comes from in vitro experiments showing that intracortical synaptic excitation is depressed after repetitive electrical stimulation (Abbott et al., 1997), and from a number of pharmacological findings. First, antagonists to presynaptic glutamate autoreceptors that mediate excitatory synaptic depression reduce extracellularly measured adaptation effects (McLean and Palmer, 1996). Second, GABA antagonists have little effect on adaptation (DeBruyn and Bonds, 1986; Vidyasagar, 1990; McLean and Palmer, 1996). Taken together, these observations support the view that adaptation is caused by a decrease in excitation rather than by an increase in inhibition. In principle this decrease could originate from a reduction in synaptic efficacy (Nelson et al., 1996; Todorov et al., 1997).

There are however a number of difficulties about our model that need to be resolved. First, adaptation may operate differently in different species. For instance, it appears to be stronger in the cat than in the monkey (Sclar et al., 1989). Second, there may be cells in which pattern adaptation reduces the firing rate responses without causing the hyperpolarization that we commonly observe (Ahmed et al., 1997). Third, it is not clear what portion of the hyperpolarization should be ascribed to synaptic effects as opposed to intrinsic cellular properties. In particular, the results of a recent study of adaptation in cat and ferret suggest that the hyperpolarization is partly caused by the activation of a $\mathrm{Na}^{+}$-dependent $\mathrm{K}^{+}$current, with the possible contribution of an electrogenic $\mathrm{Na}^{+} / \mathrm{K}^{+}$pump (McCormick et al., 1998). Fourth, the evidence in favor of adaptation changing the mutual interactions between groups of neurons is scarce at best. The results on the increase of cross-orientation suppression following adaptation to plaids are based on a very small sample, and do not allow us to know whether this behavior is prevalent or rare. Furthermore, these results were not replicated when the plaids were composed of gratings having different spatial frequencies rather than different orientations (Carandini et al., 1997a), suggesting that the increase in mutual suppression may not be a general principle. Fifth, the results of a psychophysical study of adaptation to gratings and plaids on contrast detection threshold (Foley and Chen, 1997) seem to be at odds with the model. According to Foley and Chen's analysis, which is based on a particular model of detection and masking, adaptation would indeed strengthen the suppression between units, but not necessarily in a specific fashion. The inhibitory links between units tuned to horizontal stimuli and units tuned to vertical stimuli would be strengthened by adaptation whether the adapting stimulus is a vertical grating, or a horizontal grating, or the plaid obtained by summing the two.

Further experimental data-most likely obtained with simultaneous recordings from different groups of cells - are doubtlessly needed to overcome these difficulties. Nonetheless, it is encouraging to observe a tentative agreement between the physiological findings on adaptation and a theoretical model of how the cerebral cortex should best handle information.

\section{Acknowledgements}

This work was supported by the Howard Hughes Medical Institute, and by NIH grants EY02017 to J. Anthony Movshon, and EY04726 to David Ferster. We thank H.B. Barlow for stimulating discussions.

\section{References}

Abbott, L.F., Varela, J.A., Sen, K., Nelson, S.B., 1997. Synaptic depression and cortical gain control. Science 275, 220-224.

Ahmed, B., Allison, J.D., Douglas, R.J., Martin, K.A.C., 1997. An intracellular study of the contrast-dependence of neuronal activity in cat visual cortex. Cereb. Cortex 7, 559-570.

Albrecht, D.G., Farrar, S.B., Hamilton, D.B., 1984. Spatial contrast adaptation characteristics of neurones recorded in the cat's visual cortex. J. Physiol. (London) 347, 713-739.

Albrecht, D.G., Hamilton, D.B., 1982. Striate cortex of monkey and cat: contrast response function. J. Neurophysiol. 48, 217-237.

Allison, J.D., Martin, K.A.C., 1997. Contrast adaptation produced by null direction and cross orientation stimulation of neurons in cat visual cortex. Soc. Neurosci. Abst. 23, 454

Barlow, H.B., 1990. A theory about the functional role and synaptic mechanism of after-effects. In: Blakemore, C. (Ed.), Vision: Coding and Efficiency. Cambridge University Press, Cambridge, pp. $363-375$

Barlow, H.B., 1997. The knowledge used in vision and where it comes from. Phil. Trans. R. Soc. B 352, 1143-1149.

Barlow, H.B., Földiák, P., 1989. Adaptation and decorrelation in the cortex. In: Durbin, R., Miall, C., Mitchison, C. (Eds.), The Computing Neuron. Addison-Wesley, Workingham, pp. 54-72. 
Blakemore, C., Campbell, F.W., 1968. Adaptation to spatial stimuli. J. Physiol. (London) 200, 11P-13.

Bonds, A.B., 1989. Role of inhibition in the specification of orientation selectivity of cells in the cat striate cortex. Vis. Neurosci. 2, $41-55$.

Bonhoeffer, T., Grinvald, A., 1991. Iso-orientation domains in cat visual cortex are arranged in pinwheel-like patterns. Nature 353, $429-431$.

Carandini, M., Barlow, H.B., O'Keefe, L.P., Poirson, A.B., Movshon, J.A., 1997a. Adaptation to contingencies in macaque primary visual cortex. Proc. R. Soc. London B 352, 1149-1154.

Carandini, M., Ferster, D., 1997. A tonic hyperpolarization underlying contrast adaptation in cat visual cortex. Science 276, 949-952.

Carandini, M., Heeger, D.J., Movshon, J.A., 1997b. Linearity and normalization in simple cells of the macaque primary visual cortex. J. Neurosci. 17, 8621-8644.

De Valois, K.K., De Valois, R.L., Yund, E.W., 1979. Responses of striate cortex cells to grating and checkerboard patterns. J. Physiol. (London) 291, 483-505.

DeBruyn, E.J., Bonds, A.B., 1986. Contrast adaptation in the cat is not mediated by GABA. Brain Res. 383, 339-342.

Efron, B., Tibshirani, R.J., 1991. Statistical data analysis in the computer age. Science 253, 390-395.

Földiák, P., 1990. Forming sparse representations by local anti-Hebbian learning. Biol. Cybern. 64, 165-170.

Foley, J.M., Chen, C.C., 1997. Analysis of the effect of pattern adaptation on pattern pedestal effects: a two-process model. Vis. Res. 37, 27792788.

Giaschi, D., Douglas, R., Marlin, S.G., Cynader, M.S., 1993. The time course of direction-selective adaptation in simple and complex cells in cat striate cortex. J. Neurophysiol. 70, 2024-2034.

Gilinsky, A.S., 1968. Orientation-specific effects of patterns of adapting light on visual acuity. J. Opt. Soc. Am. 58, 13-18.

Gizzi, M.S., Katz, E., Schumer, R.A., Movshon, J.A., 1990. Selectivity for orientation and direction of motion of single neurons in cat striate and extrastriate visual cortex. J. Neurophysiol. 63, 15291543.

Graham, N.V.S., 1989. Visual Pattern Analyzers. Oxford University Press, New York.

Hammond, P., Pomfrett, C.J.D., Ahmed, B., 1989. Neural motion after-effects in the cat's striate cortex: orientation selectivity. Vis. Res. 29, 1671-1683.

Heeger, D.J., 1992. Normalization of cell responses in cat striate cortex. Vis. Neurosci. 9, 181-197.

Jagadeesh, B., Wheat, H.S., Kontsevich, L.L., Tyler, C.W., Ferster, D., 1997. Direction selectivity of synaptic potentials in simple cells of the cat visual cortex. J. Neurophysiol. 78, 2772-2789.

Kisvárday, Z., Eysel, U.T., 1993. Functional and structural topography of horizontal inhibitory connections in cat visual cortex. Eur. J. Neurosci. 5, 1558-1572.

Kisvárday, Z.F., Kim, D., Eysel, U.T., Bonhoeffer, T., 1994. Relationship between lateral inhibitory connections and the topography of the orientation map in cat visual cortex. Eur. J. Neurosci. 6, 1619-1632.

Kisvárday, Z.F., Toth, E., Rausch, M., Eysel, U.T., 1997. Orientation-specific relationship between populations of excitatory and inhibitory lateral connections in the visual cortex of the cat. Cereb. Cortex 7, 605-618.
Levitt, J.B., Kiper, D.C., Movshon, J.A., 1994. Receptive fields and functional architecture of macaque V2. J. Neurophysiol. 71, 2517-2542.

Maffei, L., Berardi, N., Bisti, S., 1986. Interocular transfer of adaptation after-effect in neurons of area 17 and 18 of split chiasm cats. J. Neurophysiol. 55, 966-976.

Maffei, L., Fiorentini, A., Bisti, S., 1973. Neural correlate of perceptual adaptation to gratings. Science $182,1036-1038$.

Marlin, S.G., Hasan, S.J., Cynader, M.S., 1988. Direction-selective adaptation in simple and complex cells in cat striate cortex. J. Neurophysiol. 59, 1314-1330.

McCormick, D.A., Sanchez-Vives, M.V., Nowak, L.G., 1998. Role of membrane properties in the generation of contrast adaptation in the visual cortex. Invest. Ophthalmol. Vis. Sci. 39, 238.

McLean, J., Palmer, L.A., 1996. Contrast adaptation and excitatory amino acid receptors in cat striate cortex. Vis. Neurosci. 13, 1069-1088.

Morrone, M.C., Burr, D.C., Maffei, L., 1982. Functional implications of cross-orientation inhibition of cortical visual cells. I. Neurophysiological evidence. Proc. R. Soc. London B 216, 335354.

Movshon, J.A., Adelson, E.H., Gizzi, M.S., Newsome, W.T., 1986. The analysis of moving visual patterns, Exp. Brain Res. Suppl. II: Pattern Recognition Mechanisms, 117-151.

Movshon, J.A., Lennie, P., 1979. Pattern-selective adaptation in visual cortical neurones. Nature 278, 850-852.

Nelson, S.B., Varela, J.A., Sen, K., Abbott, L.F., 1996. Synaptic decoding of visual cortical EPSCs reveals a potential mechanism for contrast adaptation. In: Bower, J. (Ed.), The Neurobiology of Computation. Kluwer, Boston.

Ohzawa, I., Sclar, G., Freeman, R.D., 1982. Contrast gain control in the cat visual cortex. Nature 298, 266-268.

Ohzawa, I., Sclar, G., Freeman, R.D., 1985. Contrast gain control in the cat visual system. J. Neurophysiol. 54, 651-665.

Pantle, A., Sekuler, R., 1968. Size-detecting mechanisms in human vision. Science 162, 1146-1148.

Saul, A.B., Cynader, M.S., 1989a. Adaptation in single units in visual cortex: the tuning of aftereffects in the spatial domain. Vis. Neurosci. 2, 593-607.

Saul, A.B., Cynader, M.S., 1989b. Adaptation in single units in visual cortex: the tuning of aftereffects in the temporal domain. Vis. Neurosci. 2, 609-620.

Sclar, G., Lennie, P., DePriest, D.D., 1989. Contrast adaptation in the striate cortex of macaque. Vis. Res. 29, 747-755.

Shapley, R.M., Enroth-Cugell, C., 1984. Visual adaptation and retinal gain controls. Prog. Retinal Res. 3, 263-346.

Shou, T., Li, X., Zhou, Y., Hu, B., 1996. Adaptation of visually evoked responses of relay cells in the dorsal lateral geniculate nucleus of the cat following prolonged exposure to drifting gratings. Vis. Neurosci. 13, 605-613.

Todorov, E.V., Siapas, A.G., Somers, D.C., Nelson, S.B., 1997. Modeling visual cortical contrast adaptation effects. In: Bower, J. (Ed.), The Neurobiology of Computation. Kluwer, Boston.

Vautin, R.G., Berkeley, M.A., 1977. Responses of single cells in cat visual cortex to prolonged stimulus movement: neural correlates of visual after effects. J. Neurophysiol. 40, 1051-1065.

Vidyasagar, T.R., 1990. Pattern adaptation in cat visual cortex is a cooperative phenomenon. Neuroscience 36, 175-179. 